\title{
Pixel Dark Current Calibration Method to Reduce Dark Fixed Pattern Noise in Amorphous Silicon Bolometer-type Uncooled Infrared Image Sensor
}

\author{
Sang-Hwan Kim, ${ }^{1}$ Jimin Lee, ${ }^{1}$ Hyeunwoo Kwen, ${ }^{1}$ \\ Jae-Hyoun Park, ${ }^{2}$ Kyoung-Il Lee, ${ }^{2}$ and Jang-Kyoo Shin ${ }^{1 *}$ \\ ${ }^{1}$ School of Electronics Engineering, Kyungpook National University, 80 Daehak-ro, Buk-gu, Daegu 41566, Korea \\ ${ }^{2}$ Korea Electronics Technology Institute, 25 Saenari-ro, Bundang-gu, Seongnam-si, Gyeonggi-do 13509, Korea
}

(Received May 12, 2020; accepted August 13, 2020)

Keywords: infrared image sensor, bolometer, amorphous silicon, dark fixed pattern noise, 2D infrared image

In this paper, we propose a pixel dark current calibration method to reduce dark fixed pattern noise in an amorphous silicon bolometer-type uncooled infrared image sensor. This dark current calibration method utilizes an active pixel, a reference pixel, and a calibration circuit. The active and reference pixels were fabricated entirely from amorphous silicon. Even if amorphous silicon components with the same resistance are manufactured, there may be variations in resistance owing to process deviations during the manufacture of the amorphous silicon pixels. Dark fixed pattern noise due to resistance variations causes non-uniformity among the output voltages of the pixels. The operating principle of the pixel dark current calibration method to reduce dark fixed pattern noise is thus based on the subtraction of the averaged dark current of the active pixel from that of the reference pixel. The proposed pixel dark current calibration method is implemented by a chip consisting of an amorphous silicon bolometer pixel array, a calibration circuit, and column readout circuits. The entire chip was fabricated using a $0.11 \mu \mathrm{m}$ CMOS image sensor process and its performance was evaluated.

\section{Introduction}

Recently, amorphous silicon bolometer-type uncooled infrared image sensors have been developed in various fields for thermal imaging systems, night vision, surveillance, and medical imaging. ${ }^{(1-7)}$ Amorphous silicon bolometer-type uncooled infrared image sensors have many advantages owing to their low cost, low power consumption, and easy integration. However, further advances are required in terms of pixel size and device weight. Among the various uncooled infrared image sensors, the bolometer-type sensor based on micromachining technology has a higher temperature coefficient of resistance than similar sensors. ${ }^{(8-10)}$ In general, the temperature coefficient of resistance of a semiconductor is smaller than that of a metal. Therefore, a bolometer sensor that is used for manufacturing an uncooled infrared image sensor based on amorphous silicon has a large variation in output current because of heat 
generation. Furthermore, a general infrared sensor is based on the principle of thermal radiation absorption from a target object without absorbing the reflected photons that are returned to the target object. Therefore, bolometer-type uncooled infrared image sensors generally have a wider detection range from X-ray wavelengths to millimeter-order wavelengths than an infrared photon detector.

One of the important problems in the pixel arrangement of an amorphous silicon bolometer-type uncooled infrared image sensor is that process variations can occur among similarly fabricated resistors integrated in the pixel. Various methods have been studied to solve the problem of variations among bolometer resistors integrated in active and reference pixels. ${ }^{(11-17)}$ Among previously reported research, the averaging pixel current adjustment technique has been used to reduce process variations among resistors. ${ }^{(18)}$ However, a standard CMOS process was used to design the active and reference pixels with polysilicon resistors in previous research. From a technical viewpoint, it is quite similar to the research of calibrating random noise of polysilicon. ${ }^{(18)}$ However, research using polysilicon resistors was considered to be a verification of a sample calibration technique inside the chip level. ${ }^{(18)}$ In the previous research, the signal current due to the incident infrared ray could not be directly detected, because the pixel was manufactured by using the polysilicon resistor. ${ }^{(18)}$ Therefore, it is important to verify that the dark current calibration method can be applied to actual amorphous silicon bolometer-type uncooled infrared image sensors. In this paper, an uncooled infrared image sensor was fabricated using an actual amorphous silicon bolometer. Therefore, the signal current due to the incident infrared ray could be directly detected.

To compensate for the process variations in the resistors among the amorphous-silicon bolometer-type uncooled infrared image sensor pixels, a method of calibration by averaging the dark current generated in the reference pixel is proposed. The amorphous silicon bolometer resistors in the reference and active pixels have dark current components. To verify the proposed dark current calibration method, the output nodes of all the reference pixels are connected. Consequently, the output currents of the reference pixels in which the light-receiving portions are blocked to avoid the effect of incident light are averaged. The current from each active pixel array is thus subtracted from the averaged current of the reference pixel to remove the dark current of the active pixels. The dark current calibration method therefore utilizes an active pixel, a reference pixel, a calibration circuit, and a column readout circuit. An entire chip used to implement the dark current calibration method was fabricated using a $0.11 \mu \mathrm{m}$ 1-poly 4-metal CMOS image sensor (CIS) process, and its characteristics were analyzed. The CIS process is the process used to make the photodiode. However, we fabricated a bolometer using a postprocess on a chip manufactured using a CMOS process. Therefore, when manufacturing the bolometer, if the metal layer used for the chip is formed with multiple layers, it is difficult to manufacture the bolometer when the height of the chip itself is large. Therefore, among the processes, the CIS process was used because it had the smallest number of metal layers.

\section{Principle of Pixel Dark Current Calibration Method}

A schematic of the pixel dark current calibration method and its modeling are shown in Fig. 1. Each unit pixel is composed of an active pixel, a reference pixel, and a calibration circuit. The 


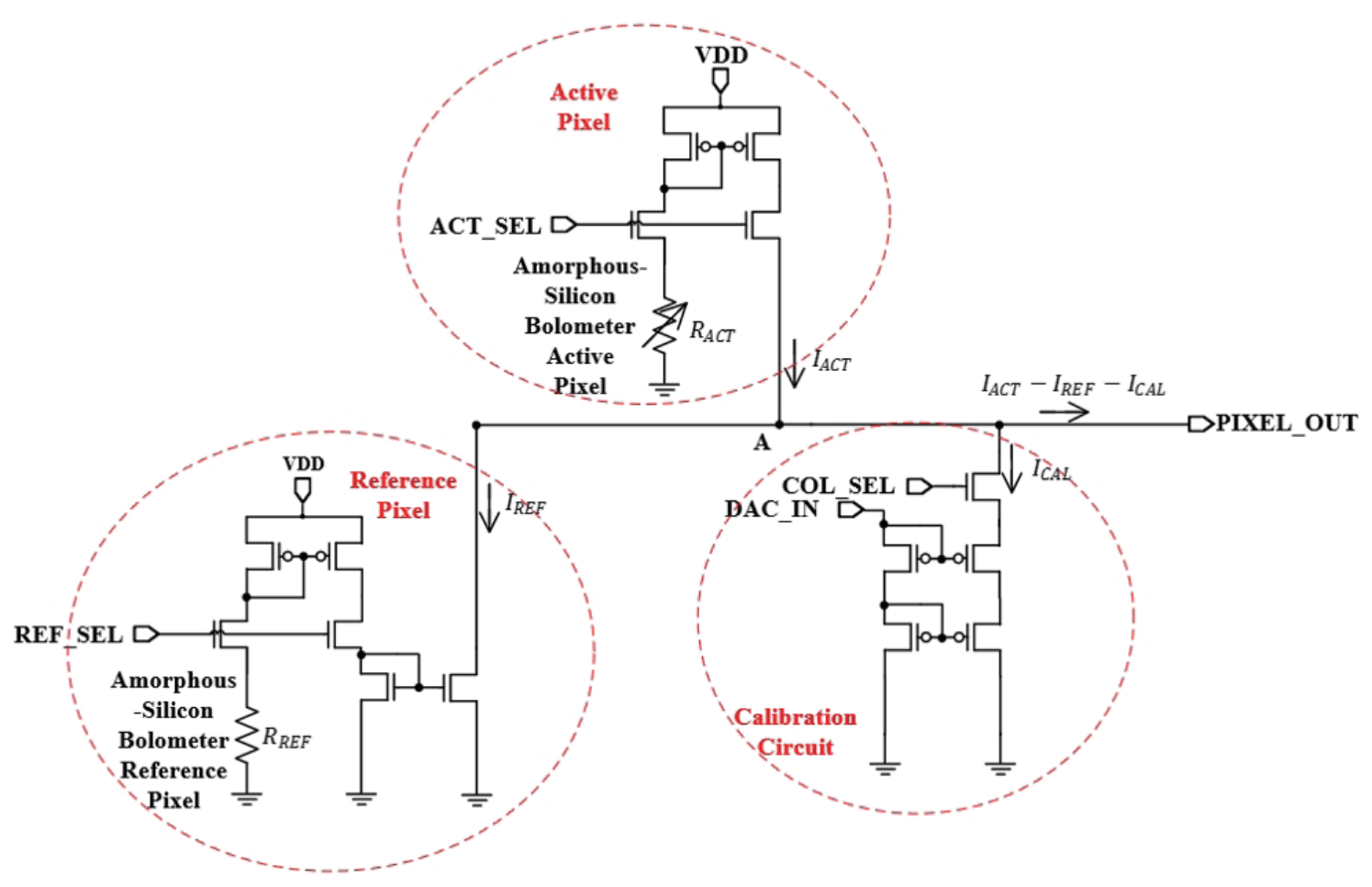

Fig. 1. (Color online) Schematic of pixel dark current calibration method.

difference between the dark current generated in each active pixel and the averaging dark current generated by the reference pixel array is computed. The active and reference pixels of the proposed image sensor were manufactured using an amorphous silicon bolometer. The amorphous silicon bolometer is a kind of resistor, and when exposed to infrared lights, the resistance value of the bolometer is changed. When Kirchhoff's current law is applied to node A, the output current of the active pixel $\left(I_{A C T}\right)$ is subtracted from that of the reference pixel $\left(I_{R E F}\right)$. To subtract the additional current, the dark current component is reduced by controlling the output current of the calibration circuit $\left(I_{C A L}\right)$. At this time, if the current of the active pixel is designed to be greater or less than the current of the reference pixel, the current direction of the calibration circuit can be determined. Through this current calculation, the dark current difference between the active and reference pixels $\left(I_{A C T}-I_{R E F}\right)$ will have information of the deviation due to the resistance variation of the active pixel. From the operation principle of the bolometer, the signal current was generated from the incident infrared light, since $I_{R E F}$ and $I_{A C T}$ were calculated as resistance. Therefore, $I_{A C T}-I_{R E F}$ reflects the resistance variation of an amorphous-silicon resistor. The relationships for calibrating the dark current using the output currents of the active and reference pixels are as follows.

$$
\begin{gathered}
I_{R E F} \propto 1 / R_{R E F}, I_{A C T} \propto 1 / R_{A C T} \\
I_{A C T}+\Delta I_{A C T} \propto 1 /\left(R_{A C T}+\Delta R_{A C T}\right) \\
I_{A C T}+\Delta I_{A C T}-I_{R E F}-I_{C A L} \triangleq \mathrm{nA} \text { order }
\end{gathered}
$$


Here, $R_{A C T}$ is the resistance of the amorphous-silicon bolometer active pixel and $R_{R E F}$ is the resistance of the amorphous silicon bolometer reference pixel. In a unit pixel, the output dark current of the reference pixel $\left(I_{R E F}\right)$ is inversely proportional to the pixel resistance $\left(R_{R E F}\right)$, and the output dark current of the active pixel $\left(I_{A C T}\right)$ is inversely proportional to the active pixel resistance $\left(R_{A C T}\right) . \quad \Delta R_{A C T}$ refers to the resistance of the amorphous silicon bolometer in the active pixel owing to a process deviation, and $\triangle I_{A C T}$ is the deviation of the output dark current of the active pixel. Therefore, $\Delta I_{A C T}$ is considered as the cause of the dark fixed pattern noise. In addition, by reducing $\Delta I_{A C T}$ using the current of the calibration circuit $\left(I_{C A L}\right)$, the final output current can be reduced to the order of nanoamperes. To adjust the calibration current for further minute current subtraction, the output current of the integrated calibration circuit $\left(I_{C A L}\right)$ based on the P-channel metal-oxide-semiconductor (PMOS) current mirror structure is controlled by the output voltage of the digital-to-analog converter (DAC_IN).

Figure 2 shows a block diagram of the pixel dark current calibration method, whose setup consists a current-to-voltage converter (CVC), an analog-to-digital converter (ADC), a frame memory, a digital-to-analog converter (DAC), an amorphous silicon bolometer-type active pixel array, and a reference pixel array. $I_{A C T}-I_{R E F}$ contains the information of pixel deviation, which is converted into a voltage through the CVC and then output as a digital value through the ADC. The digital output value having the information of pixel deviation is stored in the frame memory, and additional calculations are performed to determine the digital input value of the DAC. Thereafter, a calibration current $\left(I_{C A L}\right)$ corresponding to the output value of the DAC is generated, and it is added to or subtracted from $I_{A C T}$. At this time, if the digital-to-analog output value is controlled such that $I_{C A L}$ is almost equal to $I_{A C T}-I_{C A L}$, the pixel current offset due to the bolometer resistance variation of each active pixel can be eliminated. In practice, $I_{C A L}$ is adjusted by considering the characteristics of the CVC so that the final output current offset of each pixel is in nanoamperes.

Figures 3(a) and 3(b) show a block diagram and schematic diagram of the averaging current generator using the amorphous silicon bolometer-type reference pixel, respectively. The reason why all output nodes of the reference pixel are tied and averaged is as follows. To obtain the deviation information based on the resistance of the active pixel using the proposed calibration



Fig. 2. Modeling of pixel dark current calibration method. 


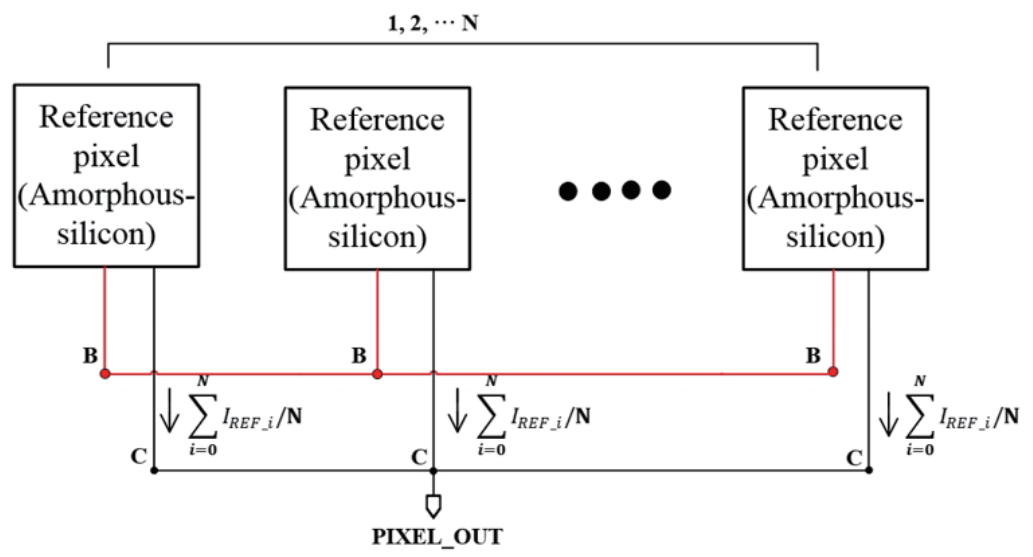

(a)

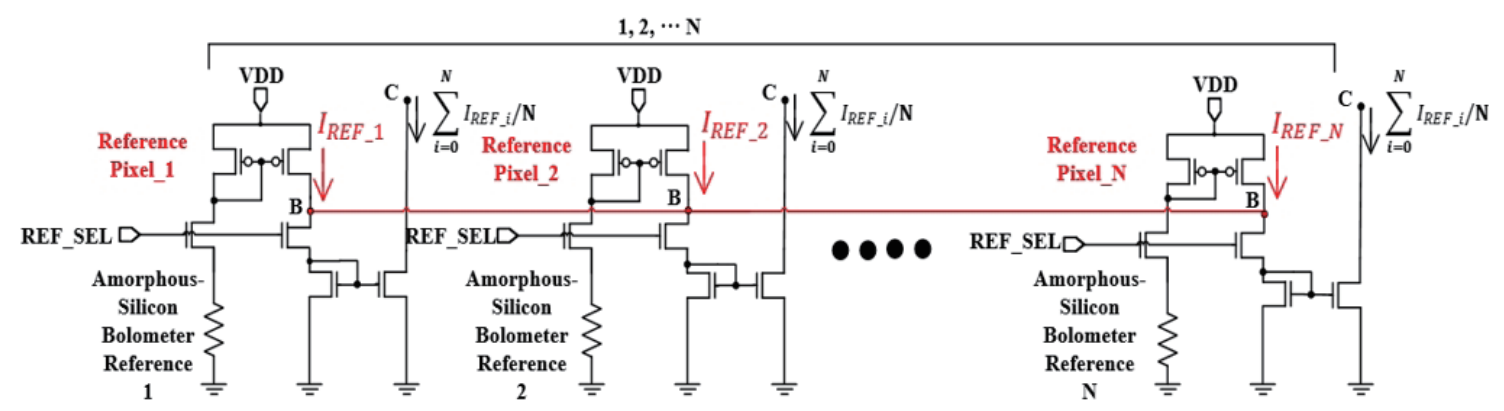

(b)

Fig. 3. (Color online) (a) Block diagram of averaging current generator using amorphous-silicon bolometer-type reference pixel. (b) Schematic of averaging current generator using amorphous-silicon bolometer-type reference pixel.

method, the resistance of the reference pixel must be fixed without any variation. However, not only the bolometer of the active pixel but also that of the reference pixel has a process deviation. Therefore, to remove the variation of the bolometer resistance of the reference pixel, all output nodes are tied. When the output nodes of all reference pixels are shared, the output current is averaged even if a resistance variation occurs in each of the reference pixels, so that the resistance variation can be canceled. The current $\left(I_{R E F_{-} I}\right)$ generated by the bolometer resistor fabricated in the unit reference pixel is output through a PMOS current mirror. Thereafter, the output nodes of all reference pixel arrays (node B) are shared to generate the sum current $\left(\sum I_{R E F_{-} I}\right)$. Finally, the average current $\left(\sum I_{R E F_{-} I} / N\right)$ is output by redistributing it to each reference pixel using an additional NMOS current mirror. The final generated average current $\left(\sum I_{R E F_{-} I} / N\right)$ is distributed equally in all reference pixels, thereby reducing the current offset generated in the active pixel.

Figure 4(a) shows a block diagram of the entire chip and Fig. 4(b) shows the layout of the entire chip. The chip circuitry is composed of a frame memory, a DAC, a calibration circuit, an active pixel, a reference pixel, a column readout circuit (integrator and CVC), and an ADC. Frame memory is integrated into the D-flip-flop and is used as the input voltage of the DAC. The DAC is used to determine the output current of the calibration circuit. The column readout circuit is composed of a current buffer, switches for selecting the signal, an integrator for 


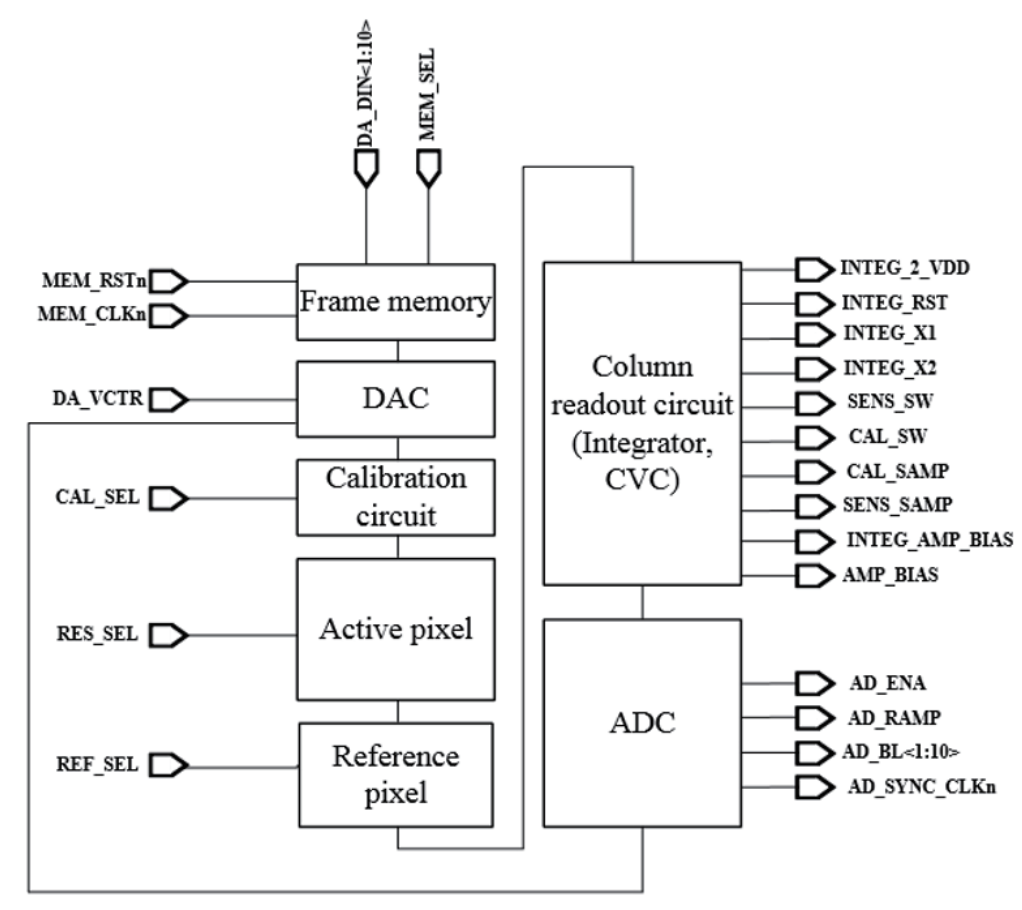

(a)

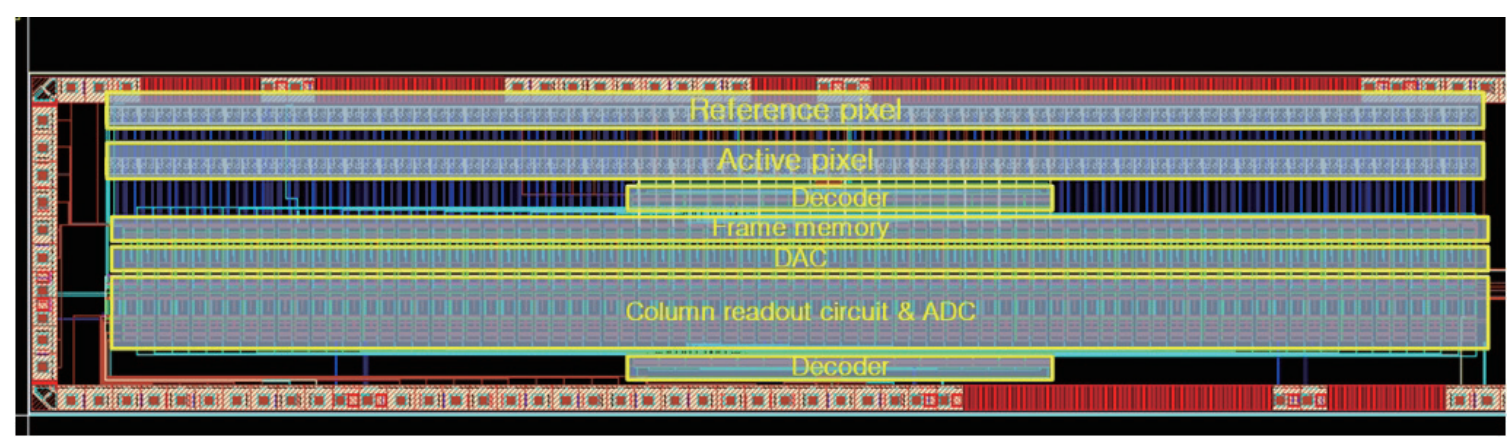

(b)

Fig. 4. (Color online) (a) Block diagram of the entire chip. (b) Layout of the entire chip.

converting the signal current after calibration, and a CVC for converting the calibration current. In addition, to operate an image sensor designed in a column parallel structure, it is designed to select each column unit using a decoder. The ADC is designed on the basis of a 10-bit singleslope ADC. The chip is fabricated using a $0.11 \mu \mathrm{m}$ CIS process.

A schematic of the column readout circuit with an integrator for converting the sensing current into voltage is shown in Fig. 5. First, the sensing current $\left(I_{A C T}-I_{R E F}-I_{C A L}\right)$ without the offset causing the dark fixed pattern noise enters the input of the current buffer. Thereafter, to convert the sensing current into a voltage, an integrator based on a two-stage amplifier is used. The integrator consists of a switch to reset the signal output (INTEG_RST), a switch to control the output voltage gain (INTEG_X1, INTEG_X2), and capacitors. Finally, the sensing current that is converted to voltage is selected by the sampling switch (SENS_SAMP) and enters the input node of the ADC. 


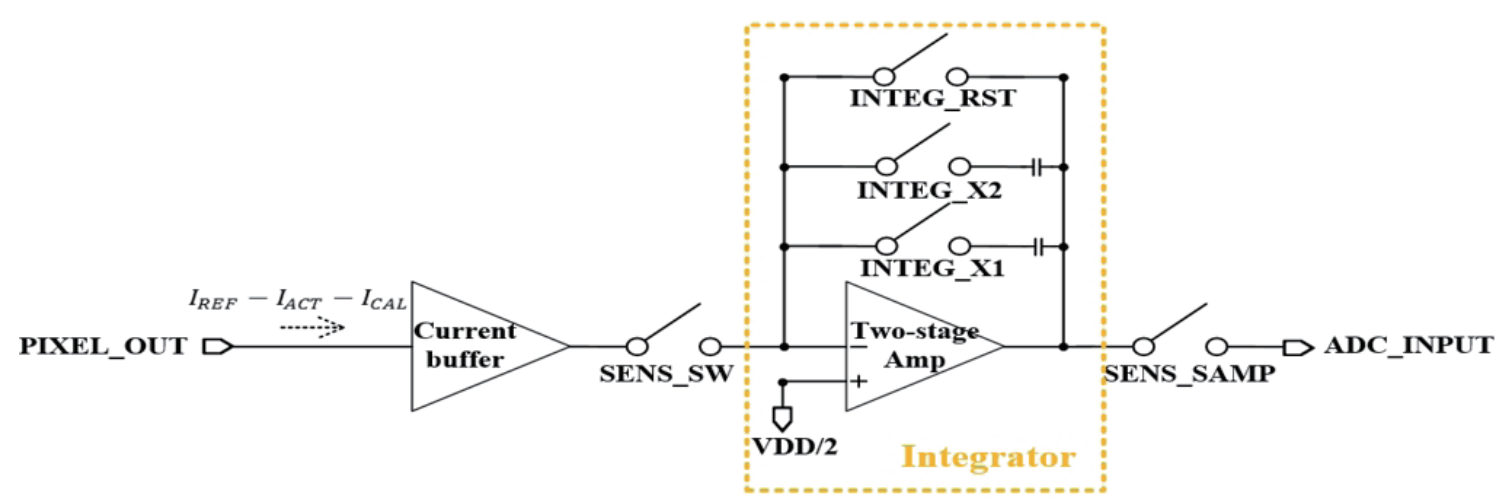

Fig. 5. (Color online) Schematic of the column readout circuit with integrator and sensing switches.

\section{Measurement Results and Discussion}

Figure 6(a) shows a photomicrograph of the active and reference pixels fabricated using amorphous silicon and Fig. 6(b) shows a scanning electron microscopy (SEM) image of the active and reference pixels. In this research, a pattern was formed on the pixel using amorphous silicon, which is generally used for the light receiving part of a bolometer-type uncooled infrared image sensor. After forming the octagonal pattern on the amorphous silicon, the generated signal electrons are input to the signal processing circuit through the metal used in the standard CMOS process. The area circled in red in Fig. 6(a) indicates a defect generated when amorphous silicon is postprocessed on a chip manufactured using a standard CMOS process.

In the proposed calibration method to remove the pixel dark current that generates dark fixed pattern noise, the most important requirement is to generate an accurate calibration current. To generate a precise calibration current, it is necessary to control even minute amounts of current. Before sensing the signal current generated in the active pixel, calibration was performed under completely dark condition. The proposed method can be applied to reduce the dark current of an image sensor. In particular, it could be effectively used to remove the fixed pattern noise caused by process variations that occur when manufacturing bolometers. The fixed pattern noise not only causes distortion of the input signal current of the practical bolometer-type uncooled infrared image sensor, but also distortion of the output image.

Figure 7 shows a graph of the calibration current plotted against the DAC by simulation. By adjusting the input digital code [the least significant bit (LSB)] of the DAC, it was confirmed that the calibration current could be increased or decreased. The input/output characteristics of the DAC were confirmed through simulation. The calibration current graph in Fig. 7 is functionalized and is represented by Eq. (4). In Eq. (4), ' $y$ ' is the calibration current, which is the $y$-axis in Fig. 7, and ' $x$ ' is the input digital code of DAC, which is the $x$-axis in Fig. 7. Therefore, Eq. (4) is the formula that stands for calibration current according to the input digital code of the DAC. The DAC used was R-2R DAC and was designed on the basis of 10 bits. The output voltage of the DAC determines the amount of output current in the calibration circuit based on the PMOS current mirror structure. Under the dark condition, as the $I_{A C T}-I_{R E F}$ value converges closer to 0 , the dark current decreases and the current generated by the incident 


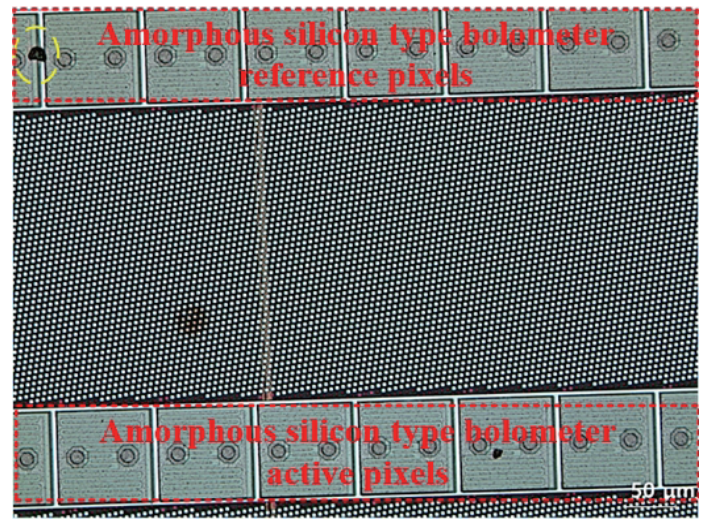

(a)

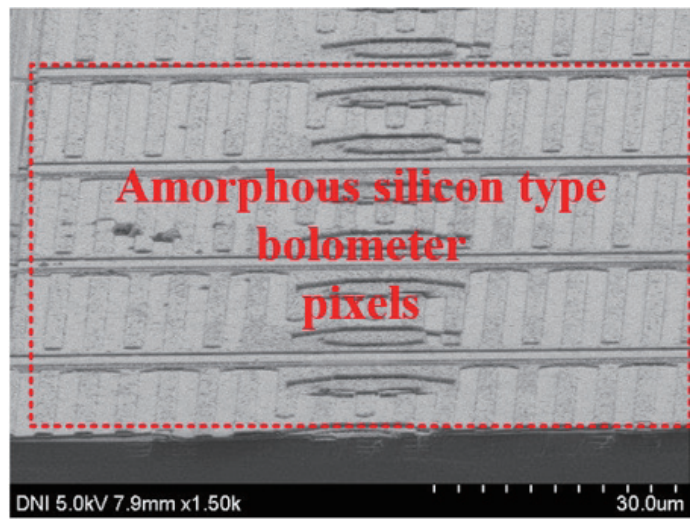

(b)

Fig. 6. (Color online) (a) Photomicrograph of active and reference pixels fabricated using amorphous silicon. (b) SEM image of active and reference pixels.

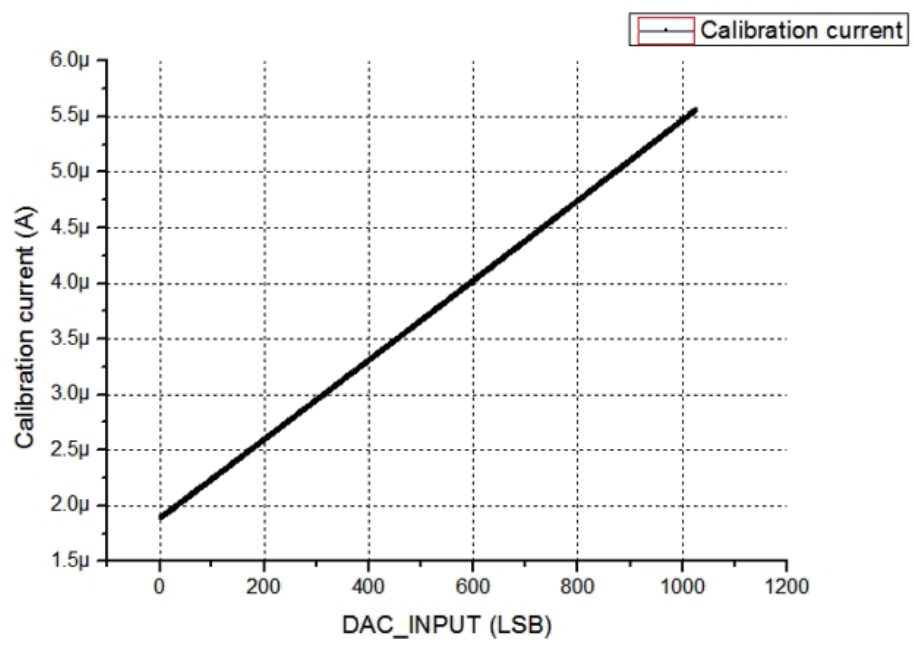

Fig. 7. (Color online) Graph of calibration current plotted against DAC input voltage by simulation.

infrared rays can be obtained. Therefore, it is important to determine the amount of calibration current $\left(I_{C A L}\right)$ in order to further subtract the $I_{A C T}-I_{R E F}$ value. The amount of calibration current generated by increasing the input LSB of the 10-bit DAC is about 3.54 nA. Finally, variation occurring in each active pixel was eliminated by adjusting the DAC input LSB value.

$$
y=\left(3.584 \cdot 10^{-9}\right) x+1.887 \cdot 10^{-6}
$$

Two-dimensional images produced by the fabricated infrared image sensor before and after applying the calibration method are shown in Figs. 8(a), and 8(b), respectively. The actual pixel array structure of the fabricated chip is $78(\mathrm{~V}) \times 1(\mathrm{H})$. The output image was acquired using a manufactured infrared image sensor having a $1 \mathrm{D}$ array structure and then represented in $2 \mathrm{D}$ form. When the image was acquired using the 10-bit single-slope ADC in the column readout 


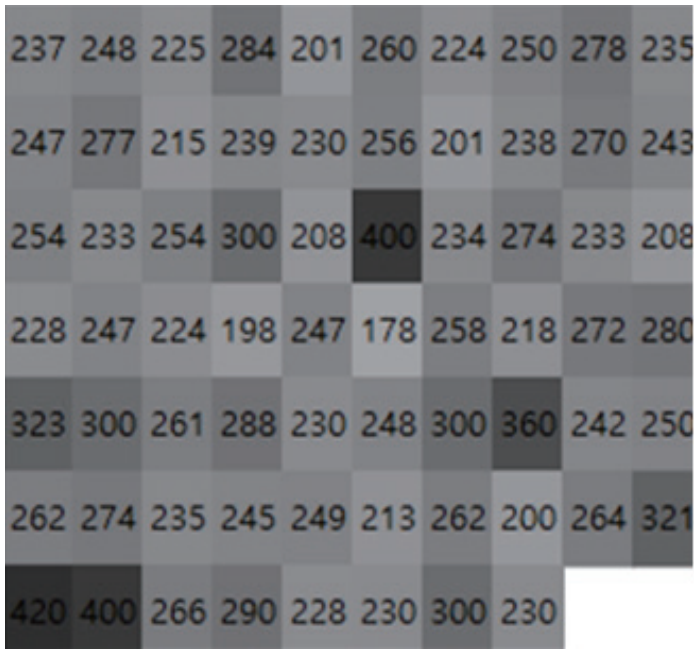

(a)



(b)

Fig. 8. Two-dimensional images produced by the fabricated infrared image sensor (a) before and (b) after applying the calibration method.

circuit, the image was acquired after removing LSB. The standard deviation of the acquired images was calculated and evaluated to assess the effect of dark fixed pattern noise on the non-uniformity of the infrared image sensor.

The actual pixel array structure of the fabricated chip is $78(\mathrm{~V}) \times 1(\mathrm{H})$. However, owing to the non-uniformity of the process of postprocessing the amorphous silicon bolometer on the fabricated chip, it was confirmed that the digital output codes of 10 pixels were output under 10. In addition, it was confirmed that the output digital code did not change according to the incident infrared light, resulting in dead pixels. Therefore, the dead pixels were not displayed in the two-dimensional image.

To verify the operation of the calibration method, the integrator output voltage of the proposed infrared image sensor was output through a 10-bit ADC. If the maximum value of digital output by 10-bit ADC is converted to a decimal number, it is 1024 . However, to remove the noise component of the ADC itself, the lower 1-bit component was removed and only 9 bits were used as the signal output. Therefore, the maximum value when converting the 9-bit ADC to a decimal number is 512, and the target digital value when applying the calibration method is 256.

Based on the digital code 256, which is half the supply voltage, the standard deviation of the image in Fig. 8(a) is 45.576. The standard deviation for Fig. 8(b) to which the calibration method was applied was $32.125 ; 45.576$ is the standard deviation of the output voltage distribution of all active pixels when the calibration method is not applied, and 32.125 is that when it is applied. The standard deviation was used to confirm the variation in the voltage distribution of the active pixel before and after applying the calibration method.

To measure the improvement of fixed pattern noise using the proposed calibration method, the output digital code of the ADC integrated inside the chip was confirmed. The lower 1 bit of the ADC output was removed. Because of the noise characteristics of the integrated 10-bit 


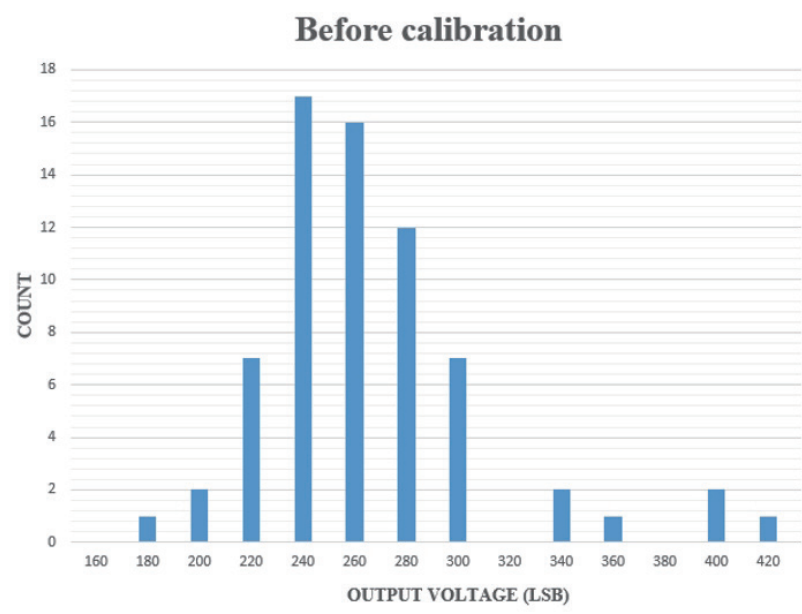

(a)

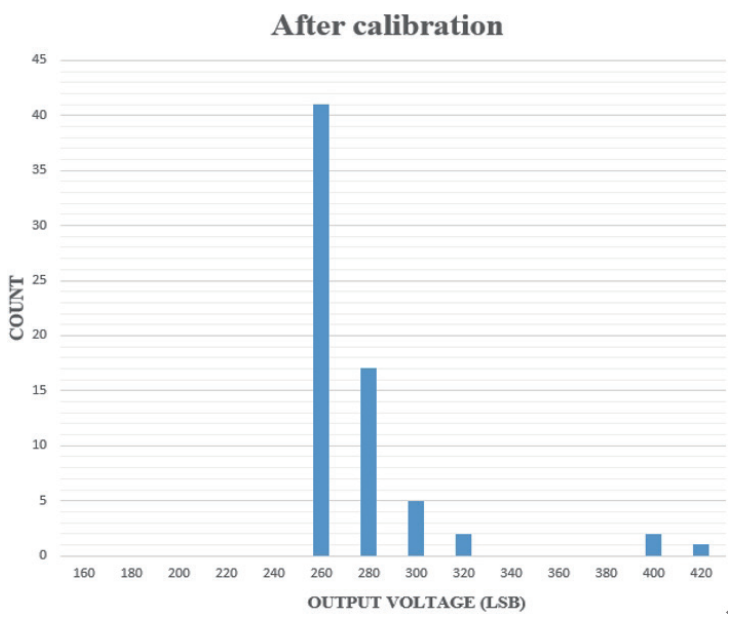

(b)

Fig. 9. (Color online) Histogram images of (a) before and (b) after applying the proposed pixel dark current calibration method to the entire array.

single slope ADC, the lower 1 bit (LSB) is not used because the output digital code occurs even when there is no input voltage. It was confirmed that the dark fixed pattern noise was about 13.5\% less than that for Fig. 8(a). Therefore, it was confirmed that the proposed calibration method can effectively remove the fixed pattern noise by comparing the standard deviations before and after the calibration.

Figures 9(a) and 9(b) show histogram images of before and after the proposed pixel dark current calibration method was applied to the entire array, respectively. It was confirmed that the pixel output voltage through the ADC before applying the calibration method was variously distributed between 180 and 400. This means that owing to variations in the amorphous silicon bolometer designed with the same resistance, variations in pixel output voltage occur under a dark condition. On the basis of the digital code 256, which is the target digital LSB voltage for calibration, 16 cases were identified before calibration, and 41 cases were identified after calibration. In particular, in the case of the pixel output voltage lower than the digital code 256, it was confirmed that the calibration method was applied to the number of all cases.

\section{Conclusions}

In this paper, we proposed a pixel dark current calibration method to reduce the dark fixed pattern noise in an amorphous silicon bolometer-type uncooled infrared image sensor. To reduce the resistance deviations causing the dark fixed pattern noise of the amorphous silicon bolometer-type uncooled infrared image sensor, the proposed pixel dark current calibration method was successfully implemented using a $0.11 \mu \mathrm{m}$ CIS process. In addition, by comparing the results before and after the calibration, it was verified that the standard deviation of the output voltage according to the resistances of the amorphous-silicon active pixel was reduced by about $13 \%$. Therefore, the experimental results confirm that the proposed method can reduce the resistance variations caused by process variations of the amorphous silicon by controlling 
the output values of the DAC. To enhance the dark fixed pattern noise reduction, there is a method of performing subtraction with a more precise output current of reference pixels by increasing the number of reference pixels. However, since the calibration current is in the nA order, the reduction in the calibration current due to parasitic resistance reduces the calibration effect. We expect that the proposed pixel dark current calibration method will be useful not only for infrared image sensors but also for noise improvement research for other types of image sensors.

\section{Acknowledgments}

This research was supported by the Industrial Core Technology Development Program (Project No. 10052933) of the Ministry of Trade, Industry \& Energy, MOTIE, the BK21 Plus project funded by the Ministry of Education, Korea (21A20131600011), and Samsung Electronics, Ltd.

\section{References}

1 S. Borri, P. Patimisco, A. Sampaolo, H. E. Beere, D. A. Ritchie, M. S. Vitiello, G. Scamarcio, and V. Spagnolo: Appl. Phys. Lett. 103 (2013) 2. https://doi.org/10.1063/1.4812438

2 A. Pesatori, A. Magnani, and M. Norgia: IEEE Int. Instrumentation and Measurement Tech. Conf. (I2MTC). (2013). https://doi.org/10.1109/I2MTC.2013.6555531

3 R. Yang, Y. He, A. Mandelis, N. Wang. X. Wu, and S. Huang: IEEE Trans. Ind. Inf. 14 (2018) 12. https://doi. org/10.1109/TII.2018.2834462

4 M. Kimata: Sens. Mater. 30 (2018) 6. https://doi.org/10.18494/SAM.2018.1802

5 W.-L. Hsu and K.-T. Chang: Sens. Mater. 31 (2019) 2. https://doi.org/10.18494/SAM.2019.2090

6 C.-D. Lee, S.-F. Tang, and T.-C. Chen: Sens. Mater. 30 (2018) 6. https://doi.org/10.18494/SAM.2018.1913

7 I. Kang, Y. A. K. Reddy, Y. B. Shin, and H. C. Lee: IEEE Sens. J. 15 (2015) 12. https://doi.org/10.1109/ JSEN.2015.2474737

8 P. Neuzil, Y. Liu, H.-H. Feng, and W. Zeng: IEEE Electron. Device Lett. 26 (2005) 5. https:10.1109/ LED.2005.846585

9 E. Iborra, M. Clement, L. V. Herrero, and J. Sangrador: J. Microelectromech. Syst. 11 (2002) 4. https://doi. org/10.1109/JMEMS.2002.800926

10 B. E. Cole, R. E. Higashi, and R. A. Wood: IEEE/LEOS Int. Cof. Optical MEMS (2000). https://doi. org/10.1109/OMEMS.2000.879627

11 X. Chen and J. Li: Int. Cof. Electrical and Control Engineering (2011). https://doi.org/10.1109/ ICECENG.2011.6058027

12 K.-D. Kim, S. Park, K.-S. Yoon, G.-G. Kang, H.-K. Han, J.-S. Choi, M.-W. Ko, J.-H. Cho, S. Lim, H.-M. Lee, H.-S. Kim, K. Lee, and G.-H. Cho: IEEE Int. Solid-Sate Circuits Conf. (ISSCC) (2018). https://doi.org/10.1109/ ISSCC.2018.8310249

13 T.-H. Yu, C.-Y. Wu, P.-Y. Chen, F.-W. Chi, J.-J. Luo, C. D. Chiang, and Y.-T. Cherng: IEEE Int. Symp. Circuits and Systems (ISCAS) (2000). https://doi.org/10.1109/ISCAS.2000.856373

14 M. Kim, S. Park, K. Lee, and H. Yoo: IEEE Photonics Technol. Lett. 30 (2018) 6. https://doi.org/10.1109/ LPT.2018.2792491

15 C. Arnaboldi and G. Pessina: IEEE Trans. Nucl. Sci. 53 (2006) 5. https://doi.org/10.1109/ NSSMIC.2005.1596284

16 D. Liu, X. Shi, G. Chen, W. Lu, and Z. Chen: IEEE Int. Conf. Electron Devices and Solid State Circuits (EDSSC), (2019). https://doi.org/10.1109/EDSSC.2019.8754397

17 P. Neuzil, J. Pekarek, V. Svatos, R. Prokop, I. Gablech, M. Pavlik, L. Fujcik, and J. Hubalek: 30th Eurosensors Conf. (EUROSENSORS) (2016). https://doi.org/10.1016/j.proeng.2016.11.327

18 S.-H. Kim, B.-S. Choi, J. Lee, J. Lee, J. Lee, J.-H. Park, K.-I. Lee, and J.-K. Shin: Sensors 19 (2019) 7. https:// doi.org/10.3390/s19071653 


\section{About the Authors}

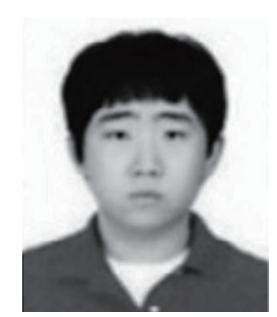

Sang-Hwan Kim received his M.S. degree in electronic engineering from Kyungpook National University, Daegu, South Korea, in 2017. He is now working toward his Ph.D. degree at Kyungpook National University. His current fields of interest are the visible \& infrared band image sensor and its readout circuits. From 2016 to 2019, he worked on uncooled infrared image sensors \& readout circuit design in Korea Electronics Technology Institute (KETI), Seongnam-si, Gyeonggi-do, South Korea. (shkim7@knu.ac.kr)

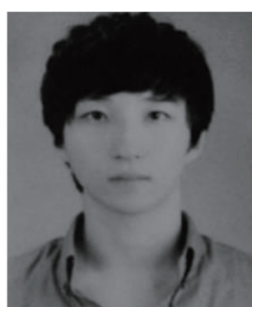

Jimin Lee received his B.S. degree in electrical engineering from Kyungil University, Gyungsan, South Korea, in 2016, and his M.S. degree in electrical engineering from Kyungpook National University, Daegu, South Korea, in 2018, where he is currently pursuing his Ph.D. degree in electrical engineering. His current research interests include CMOS image sensors and its applications. (jmLee@ee.knu.ac.kr)

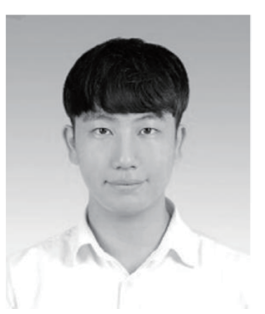

Hyeunwoo Kwen received his B.S. degree in electronic engineering from Kyungpook National University, Daegu, Korea, in 2019. He is now working toward his M.S. degree at Kyungpook National University. His current research interests include the design of the CMOS image sensor and readout circuits. (hwkwen@knu.ac.kr)

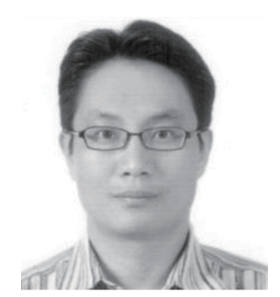

Jae-Hyoun Park received his B.S., M.S., and Ph.D. degrees in electronic engineering from Kyungoook National University, Daegu, South Korea, in 1994, 1996, and 2005, respectively. In 2004, he joined the NANO Scale Quantum Devices Research Center, KETI, Seongnam, South Korea, and he is currently a managerial research engineer. His current research interests include image sensors and readout circuits. (jhpark@keti.re.kr)

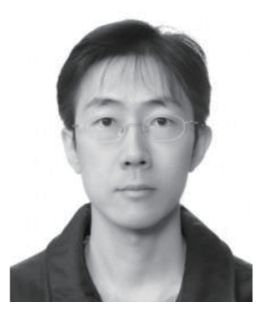

Kyoung Il Lee received his B.S. and M.S. degrees in physics from Seoul National University, Seoul, South Korea, in 1997. He is currently a managerial researcher in KETI, Seongnam, South Korea. His current research interests include the terahertz image sensor and its applications. (leeki@keti.re.kr)

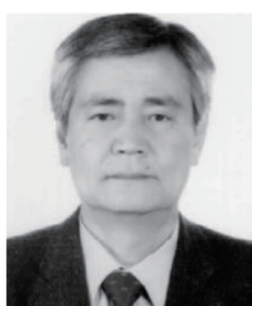

Jang-Kyoo Shin received his B.S. degree in electronic engineering from Seoul National University, Seoul, South Korea, in 1978, his M.S. degree in electrical engineering from the Korea Advanced Institute of Science and Technology, Daejeon, South Korea, in 1980, and his Ph.D. degree in electrical engineering from Colorado State University, Fort Collins, CO, USA, in 1991. He is currently a professor with the School of Electronics Engineering, Kyungpook National University, Daegu, South Korea. His current research interests include semiconductor sensors and intelligent sensor systems. (jkshin@ee.knu.ac.kr) 